\title{
Legibilidade dos termos de consentimento livre e esclarecido em ensaios clínicos
}

Lucas Lobato $^{1}$, Beatriz Santana Caçador ${ }^{2}$, Maria Flávia Gazzinelli ${ }^{3}$

\section{Resumo}

O estudo objetiva analisar a legibilidade de dois termos de consentimento livre e esclarecido (TCLE) utilizados para participação em ensaio clínico e correlacionar o grau de dificuldade dos documentos com o nível de escolaridade dos participantes. Sendo um TCLE para a elegibilidade no estudo e outro para a participação. Os TCLE foram analisados mediante utilização do índice de facilidade de leitura de Flesch (IFLF) e índice de legibilidade de Flesch-Kincaid (ILFK). Por meio da análise pelo IFLF, os TCLE para a seleção dos voluntários e participação no ensaio clínico obtiveram, respectivamente, o valor de 61 e 56 , enquanto pelo IFLK os valores foram 6,59 e 8,4, respectivamente. O TCLE para elegibilidade de voluntários e para a participação no ensaio clínico foi inadequado para $49 \%$ e $72 \%$ desses participantes. Os dois TCLE utilizados para a participação em ensaios clínicos não foram adequados para a escolaridade da maioria de seus participantes.

Palavras-chave: Bioética. Ética em pesquisa. Ensaio clínico.

\section{Resumen}

\section{La legibilidad del consentimiento en la investigación clínica}

El objetivo de este estudio es analizar la legibilidad de dos términos de consentimiento libre y aclarado (TCLA) utilizados para participar en un ensayo clínico y correlacionar el grado de dificultad de los documentos con el nivel educativo de los participantes. Siendo un TCLA para la elegibilidad en el estudio y otro para la participación. Los TCLA fueron analizados mediante utilización del índice de facilidad de lectura de Flesch Reading Ease (IFLF) Index y el Flesch-Kincaid (ILFK). A través del análisis por el IFRE, los TCLA para la selección de los voluntarios y participación en el ensayo clínico obtuvieron respectivamente, el valor de 61 y 56 , mientras por el IFLK los valores fueron 6,59 y 8,4 , respectivamente. EI TCLA para la selección de voluntarios y para la participación en el ensayo clínico fue insuficiente para el $49 \%$ y el $72 \%$ de esos participantes. Los dos TCLA utilizados para la participación en los ensayos clínicos no eran adecuados para la escolaridad de la mayoría de sus participantes. Palabras-clave: Bioética. Ética en investigación. Ensayo clínico.

\section{Abstract \\ Readability of the terms of consent in clinical research}

The aim of this study is to analyze the readability of two Informed Consent Forms (ICF) used for the participation in a clinical trial and the correlation of the degree of difficulty with the education level of the participants. The two ICF were analyzed using the Flesch Reading Ease (FRE) and the Flesch-Kincaid (FLK) readability tests. Through analysis by the Flesch score the ICF for selection of volunteers and participation in a clinical trial had, respectively, a value of 61 and 56, while the FLK values were 6.59 and 8.4 respectively. The ICF for selection and participation in the clinical trial was inadequate for $49 \%$ and $72 \%$ of these participants. Those two ICF used for participation in clinical trials were not suitable for the education of the majority of its participants. Key words: Bioethics. Ethics, research. Clinical trial.

\section{Aprovação CEP UFMG Protocolo n 01743412.3.0000.5149}

1. Mestre lucaslobato87@gmail.com 2. Mestre biacacador@gmail.com 3. Doutora flaviagazzinelli@yahoo.com.br - Escola de Enfermagem da Universidade Federal de Minas Gerais, Belo Horizonte/MG, Brasil.

Correspondência

Lucas Lobato - Escola de Enfermagem da Universidade Federal de Minas Gerais. Av. Alfredo Balena, 190, sala 508 CEP $30130-100$. Belo Horizonte/MG, Brasil. 
Os regulamentos e as diretrizes internacionais para a condução de pesquisas com seres humanos determinam que os investigadores devem obter o consentimento livre e esclarecido (CLE) dos participantes ${ }^{1}$. Em alguns países, como o Brasil, o CLE é um requisito legal e deve ser materializado em documento formal, denominado termo de consentimento livre e esclarecido (TCLE).

O TCLE é o documento que descreve a pesquisa e explica a voluntariedade na participação e permanência no estudo, devendo oferecer, de forma clara, todas as informações necessárias para possibilitar a decisão quanto à participação. As informações indispensáveis a este documento buscam conferir ao voluntário de uma pesquisa as prerrogativas para o pleno exercício de sua dignidade humana, no que tange à participação em pesquisas que envolvem seres humanos, por meio da proteção e promoção ao princípio da autonomia ${ }^{2}$.

A Resolução 196/96 do Conselho Nacional de Saúde (CNS) define que esse documento deve incluir os seguintes aspectos: justificativa da pesquisa, métodos alternativos, riscos; assistência ao sujeito da pesquisa; garantia de esclarecimentos de possíveis dúvidas do paciente durante o estudo; liberdade de desistência em qualquer fase da pesquisa; sigilo dos dados coletados e ressarcimento de despesas ou danos. É consenso na literatura que a compreensão das informações do estudo e dos direitos do participante de pesquisa pode ser garantida mediante o fornecimento de um CLE válido ${ }^{3}$. Para que se obtenha um CLE válido, torna-se indispensável que as informações aos sujeitos sejam repassadas em linguagem acessível a todos os possíveis participantes do estudo ${ }^{4}$.

Atualmente, muito se tem discutido a respeito da suficiência do TCLE para o esclarecimento e compreensão efetiva dos participantes de pesquisa. A literatura revela que muitos participantes, mesmo após a assinatura do TCLE, apresentam pouco conhecimento sobre as informações contidas no documento ${ }^{5-9}$. O nível de escolaridade dos participantes de uma pesquisa tem papel fundamental na explicação acerca do pouco conhecimento das informações do TCLE, visto que vários estudos evidenciaram associação negativa entre o nível de escolaridade e o conhecimento das informações do documento ${ }^{10-15}$. No mesmo sentido, frequentemente o TCLE pode ser considerado de difícil compreensão para vasta parcela dos participantes de pesquisa ${ }^{15-19}$.

Ao se tratar da análise da compreensão dos TCLE, merece realce um tema raramente estuda- do no Brasil: a avaliação da legibilidade dos textos desses documentos utilizados em ensaios clínicos. A avaliação dos TCLE utilizados no Brasil faz-se importante ao se considerar o perfil de escolaridade de grande parte da população, em que considerável parcela dos brasileiros é classificada analfabeta funcional ou com baixa educação formal ${ }^{15,19}$. A compreensão das informações do TCLE faz-se ainda mais importante no contexto de pesquisas clínicas por diversos motivos, tais como a complexidade das informações contidas no TCLE de investigações clínicas e os riscos associados à participação.

A complexidade dos TCLE utilizados em pesquisas clínicas decorre da própria natureza dessas investigações, tornando necessária a explicação detalhada sobre os procedimentos metodológicos das mesmas. Por conseguinte, a inclusão dessas informações pode afetar a compreensão dos documentos, haja vista que a metodologia da investigação clínica é frequentemente desconhecida dos participantes de pesquisa ${ }^{6,19-21}$. Sabe-se que os ensaios clínicos são investigações experimentais em seres humanos, envolvendo-os, inevitavelmente, em riscos proeminentes. Assim, a adequada compreensão das informações faz-se essencial, considerando que o risco associado a uma decisão deve ser proporcional à autonomia necessária para sua efetivação ${ }^{22}$.

O impasse acima referido, engendrado pela necessidade de que o participante da pesquisa compreenda o TCLE, e a dificuldade de compreensão que tradicionalmente tem sido característica desse documento tornam os ensaios clínicos um campo de interesse para o desenvolvimento de pesquisas capazes de traçar o perfil da realidade e contribuir com o processo de planejamento e obtenção do TCLE, validando sua aplicação. Ante o exposto, esta pesquisa objetiva analisar a legibilidade dos TCLE utilizados em ensaios clínicos e correlacionar o grau de dificuldade dos documentos com o perfil de escolaridade de seus participantes. O estudo também visa verificar a exigência relativa às informações que devem constar nesses documentos, de acordo com a Resolução CNS 196/96.

Ressaltamos que este artigo embasou-se nas recomendações para obtenção do TCLE para participantes de pesquisa, propostas pela Resolução CNS 196/96, considerando a atualidade de sua vigência durante a realização do presente estudo. Esta resolução, no entanto, foi revogada e substituída pela Resolução CNS 466/12, publicada no Diário Oficial da União apenas em 13 de junho de 2013. 
A nova resolução aborda de modo didático o processo de consentimento livre e esclarecido, exigindo que sejam esclarecidas aos participantes de pesquisa as mesmas informações constantes na resolução anterior.

\section{Método}

Trata-se de estudo transversal e descritivo sobre a qualidade e legibilidade de dois TCLE que deveriam ser assinados caso os voluntários desejassem participar de um ensaio clínico sobre helmintoses intestinais. O primeiro TCLE (TCLE 1) era pertinente a um estudo de elegibilidade, preliminar ao ensaio clínico, cujo objetivo era o recrutamento de potenciais participantes para a investigação clínica. Para que os voluntários pudessem ser triados para o ensaio clínico, deveriam participar desse primeiro estudo.

O segundo TCLE (TCLE 2) se referia a anuência para participação na investigação clínica denominada "Estudo duplo-cego, randomizado, controlado da tolerabilidade do consumo regular de uma mistura de óleos, em adultos residentes numa área endêmica para helmintos (ABS-00-02)" -, cujo objetivo foi avaliar a tolerabilidade de um alimento funcional, com qualidades anti-helmínticas, em adultos residentes em áreas endêmicas de helmintíases. Para que o voluntário integrasse o ensaio clínico, necessariamente deveria participar do estudo de elegibilidade e, posteriormente, assinar o TCLE do estudo clínico. Desse modo, fez-se necessária a assinatura de dois documentos para que os voluntários se inscrevessem no ensaio clínico ABS-00-02.

A pesquisa foi desenvolvida no período de agosto de 2009 a fevereiro de 2012, em Americaninhas, distrito do Município de Novo Oriente de Minas, localizado na mesorregião do Vale do Mucuri, nordeste de Minas Gerais/Brasil. O critério de inclusão foi a participação no ensaio clínico ABS-00-02, ou seja, a assinatura dos dois TCLE; enquanto o critério de exclusão foi o participante ser analfabeto, por considerar-se que não leriam os TCLE. Ressaltese que os TCLE desses participantes foram obtidos por meio da leitura desses documentos pelo pesquisador, perante uma testemunha. Na oportunidade, foram esclarecidas todas as dúvidas dos participantes. No que se refere à renda familiar, idade, raça e sexo não foi observada diferença estatisticamente significativa entre os participantes analfabetos e os que possuíam escolaridade.
Os participantes desses estudos eram homens e mulheres com idade compreendida entre 18 e 45 anos. O perfil de escolaridade dos participantes do ensaio clínico foi avaliado por um questionário estruturado, aplicado uma semana após a assinatura do TCLE do ensaio clínico ABS-00-02. No questionário, o participante era inquirido quanto a sua escolaridade formal e idade. As respostas foram registradas e tabuladas no software SPSS (Statistical Package for the Social Sciences) versão 17.0 para Windows. A quantidade de anos de estudo foi categorizada de acordo com o respectivo ano de escolaridade formal. Os dados sobre a idade dos participantes e respectiva escolaridade foram apresentados e analisados utilizando a estatística descritiva.

Os TCLE foram analisados pelo índice de facilidade de leitura de Flesch (IFLC) e pelo índice de legibilidade de Flesch-Kincaid (ILFK), ambos os métodos validados para a língua portuguesa ${ }^{16}$. Tais índices de legibilidade são modelos matemáticos que avaliam a estrutura de um texto quanto às suas frases, parágrafos e quantidade de sílabas das palavras (tamanho). A identificação dos elementos necessários para o cálculo desses índices foi operacionalizada pelo software Microsoft Word 2010.

$\mathrm{O}$ índice de facilidade de leitura de Flesch classifica o texto em escala de 100 pontos e o seu resultado classifica o texto de acordo com a dificuldade de leitura (Quadro 1). O IFLC foi calculado pela seguinte fórmula: IFLC $=206,835-[(1,015 \times$ comprimento médio da frase) $+84,6 \times$ (número médio de sílabas)].

Quadro 1. Escore Flesch distribuído de acordo com nível de escolaridade

\begin{tabular}{|c|c|}
\hline Escala Flesch & Nível de legibilidade \\
\hline $0-29$ & Muito difícil \\
$30-49$ & Difícil \\
$50-59$ & Razoavelmente difícil \\
$60-69$ & Padrão \\
$70-79$ & Razoavelmente fácil \\
$80-89$ & Fácil \\
$90-100$ & Muito fácil \\
\hline
\end{tabular}

O índice de legibilidade de Flesch-Kincaid tem sido o mais utilizado para avaliar a legibilidade de um texto e seu resultado estima os anos de estudo necessários para a adequada compreensão ${ }^{23}$ (Quadro 2). Os valores do ILFK mais efetivos são os que exigem de 6 a 10 anos de escolaridade ${ }^{16}$. Utilizou-se para o cálculo desse índice a seguinte fórmula: ILFK = 
$[(0,39 \times$ média de palavras por frase $)+(11,8 \times$ média de sílabas por palavra)] - 15,59.

Quadro 2. Classificação dos escores do índice de legibilidade de Flesch-Kincaid de acordo com os anos de escolaridade

\begin{tabular}{|c|c|}
\hline $\begin{array}{c}\text { Anos de } \\
\text { escolaridade }\end{array}$ & Equivalência escolar \\
\hline $\begin{array}{l}\text { Sem instrução e } \\
\text { menos de } 1 \text { ano }\end{array}$ & $\begin{array}{l}\text { Nunca frequentou a escola ou } \\
\text { não concluiu a } 5^{\text {a }} \text { série do ensino } \\
\text { fundamental }\end{array}$ \\
\hline 1 a 3 & $\begin{array}{l}\text { Conclusão do } 1^{\circ}, 2^{\circ} \text { ou } 3^{\circ} \text { ano do } \\
\text { ensino fundamental }\end{array}$ \\
\hline 4 a 7 & $\begin{array}{l}\text { Conclusão do } 4^{\circ}, 5^{\circ}, 6^{\circ} \text { ou } 7^{\circ} \text { ano } \\
\text { do ensino fundamental }\end{array}$ \\
\hline 8 a 10 & $\begin{array}{l}\text { Conclusão do } 8^{\circ} \text { ou } 9^{\circ} \text { ano do } \\
\text { ensino fundamental ou } 1^{\text {a }} \text { série } \\
\text { do ensino médio }\end{array}$ \\
\hline 11 a 14 & $\begin{array}{l}\text { Conclusão da } 2^{a} \text { e } 3^{a} \text { séries do } \\
\text { ensino médio ou ensino superior } \\
\text { incompleto }\end{array}$ \\
\hline 15 ou mais & $\begin{array}{l}\text { Conclusão do ensino superior ou } \\
\text { mestrado e doutorado }\end{array}$ \\
\hline
\end{tabular}

A análise da qualidade dos TCLE foi analisada pela conferência das informações requeridas pela Resolução CNS 196/96, bem como pela presença dessas informações em ambos os documentos. Nesse sentido, a qualidade do TCLE refere-se ao atendimento das informações da pesquisa exigidas por essa resolução e que devem estar presentes nesses documentos. Para tal análise, utilizou-se um roteiro para a avaliação desses documentos ${ }^{18}$ no qual os itens apontados como necessários pela Resolução CNS 196/96 fossem avaliados pelos seguintes tópicos: ausência do item; cita o item sem explicá-lo; cita e explica o item.

O presente estudo foi aprovado pelo Comitê de Ética em Pesquisa da Universidade George Washington (Washington/Estados Unidos da América) e pelo Centro de Pesquisa René Rachou (Minas Gerais), assim como o ensaio clínico ABS-00-02 e o estudo ABS-00-1, aprovados pela Comissão Nacional de Ética em Pesquisa.

\section{Resultados}

Participaram do ensaio clínico ABS-00-02 um total de 148 voluntários que, primeiramente, assinaram o TCLE 1 do estudo de elegibilidade e, posteriormente, o TCLE 2 para participação no ensaio clínico.
Desse total, 23 não participaram do estudo por serem analfabetos. Dessa forma, fizeram parte do estudo 125 indivíduos, com média de idade de 34,2 anos e mínima e máxima de 18 e 45 anos, respectivamente. A maioria dos participantes era do sexo feminino $(65,2 \%)$, casados(as) ou em união estável $(77,7 \%)$. Em relação à escolaridade dos participantes, $49 \%$ e $29 \%$ concluíram do $1^{\circ}$ ao $3^{\circ}$ ano e do $4^{\circ}$ ao $7^{\circ}$ ano do ensino fundamental, respectivamente, enquanto $13 \%$ cursaram entre o $8^{\circ}$ ano do ensino fundamental e o $1^{\circ}$ ano do ensino médio. $O$ restante dos participantes possuía formação do $2^{\circ}$ ou $3^{\circ}$ ano do ensino médio ou ensino superior incompleto/completo (9\%).

Observa-se pelo IFLF aplicado ao TCLE 2 do ensaio clínico ABS-00-02 que o grau de facilidade de leitura foi 56 , resultado que representa como razoavelmente difícil a estrutura do documento. Pelo ILFK obteve-se o valor 8,4, o que corresponde à necessidade de ter, no mínimo, oito anos de escolaridade para a compreensão adequada. Em termos de equivalência escolar, faz-se necessária a conclusão de, ao menos, o $8^{\circ}$ ano do ensino fundamental. Tendo em vista que a minoria dos participantes (22\%) possuía escolaridade acima do $8^{\circ}$ ano, esse documento foi considerado inadequado para os outros $78 \%$ dos participantes que possuíam escolaridade inferior a oito anos de escolaridade.

Com pertinência ao TCLE 1 do estudo de elegibilidade, pela avaliação do IFLF o grau de facilidade de leitura foi 61 , significando que o documento foi considerado com dificuldade "padrão". Pelo ILFK obteve-se o valor 6,59, o que corresponde à necessidade se ter, no mínimo, seis anos de escolaridade para compreender adequadamente o documento. Em termos de equivalência escolar, faz-se preciso que o sujeito tenha concluído, no mínimo, o $4^{\circ}$ ano do ensino fundamental. Considerando que $51 \%$ dos participantes possuíam escolaridade acima do $4^{\circ}$ ano, esse documento foi considerado inadequado para os outros $49 \%$ dos participantes que possuíam escolaridade inferior.

Com relação à avaliação da qualidade dos TCLE estudados de acordo com a escala utilizada neste estudo, pode-se observar que esses documentos são bem elaborados tecnicamente, tendo em vista que continham todos os elementos descritos nas resoluções CNS 466/12 e 196/96 (Quadro 3). A análise do Quadro 3 evidencia que em ambos os documentos observados a forma de ressarcimento ou indenização dos gastos ou danos ocasionados pelo estudo são apenas citados, sem a respectiva explicação desses temas. 
Quadro 3. Itens presentes na Resolução 466/12 que devem estar presentes no termo de consentimento livre e esclarecido e a presença desses itens nos TCLE dos estudos ABS-00-01 e ABS-00-02

\begin{tabular}{|c|c|c|c|c|c|c|}
\hline \multirow{2}{*}{ Item } & \multicolumn{3}{|c|}{ ABS-00-01 } & \multicolumn{3}{|c|}{ ABS-00-02 } \\
\hline & $A$ & $\mathrm{P}$ & $P+E$ & $A$ & $\mathrm{P}$ & $P+E$ \\
\hline Justificativa & & & $x$ & & & $x$ \\
\hline Objetivos & & & $x$ & & & $x$ \\
\hline Procedimentos & & & $X$ & & & $x$ \\
\hline Desconfortos e riscos & & & $x$ & & & $x$ \\
\hline Métodos alternativos & & $x$ & & & & $x$ \\
\hline Forma de acompanhamento e assistência e responsáveis & & & $X$ & & & $x$ \\
\hline Liberdade do sujeito se recusar a participar da pesquisa & & & $x$ & & & $x$ \\
\hline Garantia do sigilo & & & $\mathrm{X}$ & & & $x$ \\
\hline Formas de ressarcimento & & $x$ & & & $x$ & \\
\hline Formas de indenização & & $x$ & & & $x$ & \\
\hline Sobre a metodologia & & & $x$ & & & $x$ \\
\hline Benefícios esperados & & & $x$ & & & $x$ \\
\hline
\end{tabular}

Nota: A - Ausente; $P$ - Presente; $P+E$ - Presente e com explicação.

\section{Discussão}

Os TCLE utilizados para participação no estudo de elegibilidade e para o ensaio clínico ABS-00-02, analisados pelo IFLF e ILFK, foram considerados inadequados para grande parcela dos participantes do ensaio clínico, sendo o primeiro inadequado para voluntários com escolaridade formal inferior ao $4^{\circ}$ ano do ensino fundamental e o segundo, para participantes que não completaram o $8^{\circ}$ ano do ensino fundamental. Ante esta realidade destaca-se, entretanto, que esses documentos possuem melhor legibilidade do que os TCLE utilizados em estudos clínicos e multicêntricos realizados em São Paulo, nos quais os coeficientes de legibilidade do IFLF variaram de 32 a 44 (média aritmética: 38,5 ) e ILFK médio de 18 anos ${ }^{18}$.

A apreensão com os resultados deste estudo reside especialmente na inadequação dos dois TCLE utilizados para participação no ensaio clínico ABS00-02 ao perfil de escolaridade da maioria de seus participantes. A discrepância entre a escolaridade requerida para entender o TCLE e o perfil amostral em que esse documento foi aplicado também foi percebida em estudo realizado em Porto Alegre, no qual se observou que para a compreensão de $91,7 \%$ dos TCLE utilizados nos anos de 1998 e 1999 eram necessários 11 anos ou mais de estudo, mas apenas $16,6 \%$ da população possuía esse perfil ${ }^{23}$. Resultados semelhantes também foram encontrados em outros estudos realizados no Brasil e na França ${ }^{24,25}$.
Essa problemática torna-se potencializada quando se considera que o perfil de escolaridade dos participantes do ensaio clínico ABS-00-02 é semeIhante ao observado em outros ensaios clínicos realizados no Brasil. Observou-se que o perfil amostral de estudos com participantes de pesquisas clínicas conduzidas em hospitais públicos no Brasil ${ }^{17} \mathrm{e}$ em centros especializados em pesquisas clínicas ${ }^{26}$ conta com porcentagem semelhante de participantes que cursaram apenas o ensino fundamental. Haja vista que nesta pesquisa apenas $40 \%$ dos respondentes possuíam escolaridade adequada para o entendimento do documento, os demais provavelmente se depararam com dificuldades no entendimento das informações constantes, fato que nos permite considerá-lo como "inadequado", inviabilizando, dessa forma, a efetividade do consentimento. Sabe-se que um texto mal compreendido pode resultar em prejuízo no esclarecimento das informações, da voluntariedade e do consentimento propriamente dito ${ }^{18}$.

Além das implicações éticas na validade do CLE para os participantes do ensaio clínico ABS-00-02, deve-se ampliar essa discussão para outros contextos no Brasil. Ressalte-se que a situação evidenciada neste estudo reflete o débito educacional do país, onde $28 \%$ da população, entre 15 e 64 anos, é considerada analfabeta absoluta ou rudimentar. Dentre os que cursam ou cursaram do $6^{\circ}$ ao $9^{\circ}$ ano do ensino fundamental, $24 \%$ ainda permanecem no nível rudimentar, ou seja, conseguem ler e entender apenas frases simples e curtas, como manchetes jorna- 
lísticas, por exemplo. Dentre a população que cursou alguma série ou completou o ensino médio, apenas $41 \%$ atingem o nível pleno de alfabetização ${ }^{19}$.

Não distante dessa situação, em Minas Gerais, no recorte populacional de 18 a 24 anos, 44,5\% da população possui menos de oito anos de estudo, sendo 3,3\% analfabetos. Situação mais alarmante ocorre na população adulta (25 anos ou mais), na qual esses valores elevam-se para $67,9 \%$ e $14,8 \%$, respectivamente ${ }^{27}$. Essa conjuntura agrava-se na análise microrregional, já que em Novo Oriente de Minas, na faixa etária de 18 a 24 anos, a porcentagem de habitantes com menos de oito anos de estudo atinge $75,4 \%$, com $19,3 \%$ de analfabetos. Ao estender a discussão para a população adulta (acima de 25 anos), esses valores elevam-se para 93,2\% e $57,1 \%$, respectivamente. Com efeito, essa faixa etária, em média, apresenta dois anos de estudo ${ }^{27}$.

Frente a esse alarmante quadro, é importante refletir sobre o impacto dessa situação na condução de pesquisas clínicas realizadas no Brasil, pois muitos participantes podem não ser capazes de ler e compreender o TCLE. Esse documento, segundo a Resolução CNS 196/96, deve ser aplicado ao indivíduo da pesquisa e é conveniente que se solicite sua leitura, bem como sua explicação verbal ${ }^{4}$. A discrepância entre o perfil educacional do Brasil, em praticamente todas as suas regiões, e a escolaridade adequada para a compreensão de um TCLE traz à tona a necessidade de promover discussões e reflexões entre a comunidade científica, universidades e sociedade civil, no intuito de intervir sobre este panorama e construir vias de transformação desta realidade preocupante.

Coloca-se em discussão, dessa forma, a autenticidade do consentimento de participantes que podem não ter compreendido a pesquisa e os procedimentos que nela estão implicados, embora expressem anuência de sua participação por meio da assinatura. Legalmente, há coerência com os requisitos previstos na Resolução CNS 196/96, mas questiona-se a dimensão ética que envolve a autonomia em consentir sem compreender o que propõe a pesquisa.

Tendo em vista a fragilidade do processo educacional no Brasil, revelada no expressivo número de analfabetos no país, associada à complexidade dos termos utilizados nos TCLE, sobretudo das pesquisas clínicas, deparamo-nos com o delineamento de situações que fortalecem a lógica de dominação exercida por profissionais de saúde e pesquisadores sobre a população. Ante as determinações sociais e as desigualdades a que cotidianamente está exposta grande parte da população brasileira, questiona-se as implicações éticas de uma prática que, de forma legítima e em conformidade com as determinações legais, tem colocado pessoas em condição de subserviência, ferindo o princípio ético da autonomia. Assim, reforça-se a lógica de exercício de poder a partir do saber, um saber técnico inacessível e fora da possibilidade de compreensão de determinado grupo populacional. Por não compreenderem, não há questionamentos, reflexões e críticas, fazendo com que os sujeitos submetam suas vidas aos interesses dos pesquisadores e às determinações dos profissionais de saúde.

Essa reflexão encontra ressonância com a perspectiva foucaultiana de biopoder. Para o autor, biopoder refere-se à lógica de designação da vida a partir de sua inscrição em domínios biológicos, respaldados por um saber científico e um saber técnico que se estabelece como saber-poder, que, por sua vez, cria mecanismos de controle e dominação sobre os sujeitos ${ }^{28}$. Estabelece-se, dessa forma, relação assimétrica de poder entre o pesquisador e o participante da pesquisa. Nesta relação, o pesquisador detém a linguagem técnica sobre a pesquisa transposta no TCLE, tornando inacessível a compreensão dos participantes. $E$, perante esta limitada compreensão que os participantes possuem da pesquisa, na qual consentem em fazer parte, sua liberdade de escolha e tomada de decisão é quase inexistente. Estabelece-se, assim, uma contradição importante, pois ao mesmo tempo em que o TCLE tem por finalidade resguardar a autonomia e tomada de decisão esclarecida dos sujeitos, pode igualmente transformar-se em instrumento que legitima as estratégias de dominação sobre populações vulneradas, trazendo implicações éticas de grandes proporções.

Deve-se ressaltar que apesar das implicações da inadequação desse documento ao perfil de escolaridade dos participantes, este motivo não deve ser exclusivamente apontado como barreira na compreensão das informações do TCLE. Essa conclusão é apoiada por estudo realizado na França, apontando que a melhoria da qualidade do TCLE e a tentativa de adequação desse documento ao perfil de escolaridade dos participantes não resultou em melhoria da compreensão das informações contidas no documento ${ }^{29}$. Outro elemento estrutural avaliado neste estudo diz respeito ao fornecimento das informações necessárias a um CLE válido. Observou-se que os TCLE avaliados possuíam todas as informações descritas na Resolução CNS 196/96 como indispensáveis para um CLE válido, bem como na Resolução 466/12 ${ }^{30}$. No entanto, a disposição de todas essas informações 
não é capaz de explicar a inadequação desse documento ao perfil amostral de ensaios clínicos, vez que estudos realizados no Brasil e em países desenvolvidos assinalaram para a possibilidade de o TCLE ser completo e, ao mesmo tempo, de fácil leitura ${ }^{31-33}$.

\section{Considerações finais}

Ante os resultados deste estudo, conclui-se que o TCLE para participação em ensaios clínicos pode ser, para grande parcela de seus participantes, uma barreira para o conhecimento das informações acerca da pesquisa. Nesse sentido, esse documento pode não atingir seu papel de promover a autonomia dos participantes nos estudos, revelando uma contradição importante e que pode colocar em debate, inclusive, sua razão de existir, considerando a forma como está estruturado.

O desenvolvimento de um TCLE em diferentes níveis de legibilidade, de acordo com as respectivas escolaridades formais necessárias para sua adequada compreensão, pode ser considerado uma possibilidade para contornar essas barreiras. O desenvolvimento de intervenções educativas associadas ao CLE também desponta como possibilidade para contornar a insuficiência do TCLE para divulgação das informações. Essa estratégia tem se mostrado eficaz para que os participantes compreendam as informações da pesquisa, tais como objetivos, natureza e direitos do participante de pesquisa ${ }^{5}$. Inclusive, a Resolução CNS 466/12 apresenta o termo de consentimento de pesquisa como um processo de consentimento, e não apenas como um processo burocrático de assinatura.

Destacamos que os resultados deste estudo permanecem atuais mesmo com a revogação da Resolução CNS 196/96, haja vista que a Resolução CNS 466/12 recomenda que o participante seja esclarecido sobre os mesmos aspectos da resolução anterior. No mesmo sentido, os resultados deste estudo implicam a necessidade de considerar a novidade trazida pela nova resolução sobre ética em pesquisa, na qual, para ser livre e esclarecido, o consentimento não pode se restringir à assinatura burocrática de um documento, mas a um processo de confiança mútua entre pesquisador e voluntário.

Os resultados sugerem a necessidade de incrementar o debate acerca da finalidade do TCLE. Este documento não corresponde, portanto, a mero requisito formal que torna viável a realização de pesquisas com seres humanos. Ao contrário, responde à perspectiva ética mais ampla cujo compromisso se assenta na construção da cidadania e respeito à autonomia dos participantes.

Sendo assim, o TCLE estruturado de modo inacessível à compreensão dos participantes pode ser considerado dispositivo ${ }^{34}$ de fortalecimento das estratégias de biopoder por meio das quais se produz e se perpetua uma relação de domínio entre o pesquisador, detentor do saber e da linguagem técnica nele contidos, e o sujeito da pesquisa que tem sua autonomia e alteridade sequestradas por consentir em participar de pesquisa cuja finalidade não conseguiu compreender - o que sugere questionar a autenticidade de sua tomada de decisão.

Perante o abismo existente entre a linguagem do TCLE e a realidade social da maioria da população brasileira, emerge a necessária discussão sobre a bioética da proteção e a questão dos vulnerados. A organização social do Brasil e de grande parte dos países da América Latina é marcada por fortes desigualdades, além de restrito acesso à educação formal, tanto no que se refere aos anos de estudo quanto à qualidade do ensino proporcionado. Esta realidade determina aos sujeitos que fazem parte deste contexto vivências de situações adversas, para as quais não possuem capacidade de enfrentamento, criando determinações sociais que colocam vasta parcela da população em condição de vulneração. A vulneração é um conceito relacionado a uma condição de existência específica de grupos populacionais particulares expostos a adversidades independentemente de suas vontades, para as quais não dispõem de meios necessários para o enfrentamento. No presente trabalho, considera-se como situação de vulneração os poucos anos de estudo, o que lhes impossibilita compreender não apenas os TCLE, mas outras questões relacionadas à vida cotidiana e suas possibilidades de escolha e tomada de decisão autônoma ${ }^{35}$.

Neste estudo também se ressalta que a disposição de todas as informações descritas pelas resoluções sobre ética em pesquisa não necessariamente implicam maior dificuldade de legibilidade do TCLE. Assim sendo, destaca-se que todas essas informações devem ser apresentadas nesses documentos, mas considerando estratégias que podem facilitar seu entendimento. Como estratégia potencialmente capaz de viabilizar a compreensão mais efetiva do TCLE, citamos como exemplo a realização de intervenções educativas associadas ao processo de obtenção do consentimento livre e esclarecido, como um recurso pedagógico capaz de favorecer o conhecimento sobre as informações de um ensaio clínico ${ }^{10}$, inclusive já realizadas com efetividade na região deste estudo ${ }^{5,36}$. 
A importância dessas intervenções também se faz imperativa ao considerar a necessidade de inclusão de analfabetos em pesquisas, os quais devem também ter acesso às informações da pesquisa. As intervenções educativas associadas ao processo de obtenção do consentimento livre e esclarecido criam condições para a superação de situações de heteronomia ${ }^{37}$ e dominação dos sujeitos, possibilitando-lhes a participação de fato autônoma em pesquisas.

\section{Agradecimentos}

Agradecemos aos voluntários do ensaio clínico ABS-00-02 que se dispuseram a participar do presente estudo e a todos os colegas pesquisadores que direta ou indiretamente contribuíram para sua realização.

\section{Referências}

1. Council for International Organizations of Medical Sciences. International ethical guidelines for biomedical research involving human subjects. WHO, colaborador. Genebra: Cioms/OMS; 1993.

2. Junges JR. Exigências éticas do consentimento livre e esclarecido. Rev. bioét. (Impr.). 2007;15(1):7782.

3. Beauchamp TL, Childress JF. Princípios de ética biomédica. $4^{\mathrm{a}}$ ed. São Paulo: Loyola; 2013.

4. Brasil. Conselho Nacional de Saúde. Resolução $n^{\circ}$ 196, de 10 de outubro de 1996. Aprova diretrizes e normas regulamentadoras de pesquisas envolvendo seres humanos. [Internet]. 1996 (acesso 20 set. 2004). Disponível: http://conselho.saude.gov.br/docs/Reso196.do

5. Gazzinelli MF, Lobato L, Matoso L, Avila R, Marques RC, et al. Health education through analogies: preparation of a community for clinical trials of a vaccine against hookworm in an endemic area of Brazil. PLoS Negl Trop Dis. 2010;4(7):749-62.

6. Mandava A, Pace C, Campbell B, Emanuel E, Grady C. The quality of informed consent: mapping the landscape. A review of empirical data from developing and developed countries. J Med Ethics. 2012;38(6):356-65.

7. Kaewpoonsri N, Okanurak K, Kitayaporn D, Kaewkungwa J, Vijaykadg S, et al. Factors related to volunteer comprehension of informed consent for a clinical trial. Southeast Asian J Trop Med Public Health. 2006;37(5):996-1.004.

8. Minnies D, Hawkridge T, Hanekon W, Ehrlich R, London L, Hussey G. Evaluation of the quality of informed consent in a vaccine field trial in a developing country setting. BMC Med Ethics. 2008;9(15):1-9.

9. Chaisson LH, Kass NE, Chegeta B, Mathebula U, Samandari T. Repeated assessments of informed consent comprehension among hiv-infected participants of a three-year clinical trial in Botswana. PLoS One. 2011;6(10):e791.

10. Flory J, Emanuel E. Interventions to improve research participants' understanding in informed consent for research. Jama. 2004;292(13):1.593-601.

11. Hill Z, Tawiah-Agyemang C, Odei-Danso S, Kirkwood B. Informed consent in Ghana: what do participants really understand? J Med Ethics. 2008;34(1):48-53.

12. Moodley K, Parker M, Myer L. Informed consent and participants perceptions of influenza vaccine trials in South Africa. J Med Ethics. 2005;31(12):727-32.

13. Marshall PA. Adebamowo CA, Adeyemo AA, Ogundiran TO, Vekich M, et al. Voluntary participation and informed consent to international genetic research. Am J Public Health. 2006;96(11):1.98995.

14. Araujo DVP, Zoboli ELCP, Massad E. Como tornar os termos de consentimento mais fáceis de ler? Rev Assoc Med Bras. 2010; 56(2):151-6.

15. Lorenzo C. O consentimento livre e esclarecido e a realidade do analfabetismo funcional no Brasil: uma abordagem para a norma e para além da norma. Rev. bioét. (Impr.). 2007;15(2):268-82.

16. Goldim, JR. Consentimento e informação: a importância da qualidade do texto utilizado. Rev HCPA. 2006;26(3)117-22.

17. Meneguin S, Zoboli ELCP, Domingues, RZL, Nobre MR, Cesar LAM. Entendimento do termo de consentimento por pacientes partícipes em pesquisas com fármaco na cardiologia. Arq Bras Cardiol. 2010; 94(1):4-9.

18. Miranda VC, Fêde ABS, Lera AT, Ueda A, Antomangelo DV, et al. Como consentir sem entender? Rev Assoc Med Bras. 2009;55(3):328-34.

19. Instituto Paulo Montenegro. Indicador de alfabetismo funcional: principais resultados - Inaf Brasil 2009. [Internet]. São Paulo; 2007. (acesso 12 jan. 2012). Disponível: http://www. ibope. com.br/ipm/relatorios/relatorio_inaf_2009.pdf

20. Khalil SS, Silverman HJ, Raafat M, El-Kamary S, El-Setouhy M. Attitudes, understanding, and concerns regarding medical research amongst Egyptians: a qualitative pilot study. BMC Med Ethics. 2007;8(9):131-40.

21. Pace C, Emanuel EJ, Chuenyam T, Duncombe C, Bebchuk JD, et al. The quality of informed consent in a clinical research study in Thailand. IRB. 2005;27(1):9-17. 
22. Jansen LA. Two concepts of therapeutic optimism. J Med Ethics. 2011;37(9):563-6.

23. Schmitz EF, Cunha DID, Goldim JR. Índices de legibilidade em termos de consentimento livre e esclarecido de projetos de pesquisa do Hospital de Clínicas de Porto Alegre. III Congresso Brasileiro de Bioética e I Congresso de Bioética do Cone Sul. [anais]. Porto Alegre: UFRGS; 2000.

24. Francisconi CF, Benincasa C, Teixeira R, Bulla MC, Clotet J, Goldim JR. Termos de consentimento livre e esclarecido em uso nas áreas assistenciais do HCPA. III Congresso Brasileiro de Bioética e I Congresso de Bioética do Cone Sul. [anais]. Porto Alegre: Universidade Federal do Rio Grande do Sul; 2000.

25. Ménoni V, Lucas N, Leforestier JF, Dimet J, Doz F, et al. The readability of information and consent forms in clinical research in France. PLoS One. 2010;5(5):10.576.

26. Lacativa PGS, Szrajbman M, Silva DASM, Melazzi ACC, Gregório LH, et al. Perfil de sujeitos de pesquisa clínica em um centro ambulatorial independente. Cinc. Saúde Coletiva. 2008;13(3):1.02332.

27. Programa das Nações Unidas para o Desenvolvimento. Atlas de desenvolvimento humano IDH/2003. [Internet]. (acesso 12 dez. 2012). Disponível: http://www.pnud.org.br

28. Foucault M. Microfísica do poder. In: Machado R, organizador. Rio de Janeiro: Graal; 1979.

29. Paris A, Brandt C, Cornu C, Maison P, Thalamas C, Cracowski JL. Informed consent document improvement does not increase patients' comprehension in biomedical research. $\mathrm{Br} \mathrm{J} \mathrm{Clin}$ Pharmacol. 2010;69(3):231-7.

30. Brasil. Conselho Nacional de Saúde. Resolução $n^{\circ}$ 466, de 12 de dezembro de 2012. Aprova diretrizes e normas regulamentadoras de pesquisa envolvendo seres humanos. [Internet]. 2012 (acesso 10 jul. 2013). Disponível: http://conselho.saude.gov.br/resolucoes/2012/Reso466.pdf

31. Joffe S, Cook EF, Cleary PD, Clark JW, Weeks JC. Quality of informed consent in cancer clinical trials: a cross-sectional survey. Lancet. 2001;358(9.295):1.772-7

32. Doak CC, Doak LG, Friedell GH, Meade CD. Improving comprehension for cancer patients with low literacy skills: strategies for clinicians. CA Cancer J Clin. 1998;48(3):151-62.

33. Coyne CA, Xu R, Raich P, Plomer K, Dignan M, et al. Randomized controlled trial of an easy-to-read informed consent statement for clinical trial participation: a study of the Eastern Cooperative Oncology Group. J Clin Oncol. 2003;21(5):836-42.

34. Foucault M. História da sexualidade I: a vontade de saber. In: Albuquerque MTC, Albuquerque JAG, tradutores. 13ª ed. Rio de Janeiro: Graal; 1999.

35. Boy R, Schramm FR. Bioética da proteção e tratamento de doenças genéticas raras no Brasil: o caso das doenças de depósito lisossomal. Cad. Saúde Pública. 2009;25(6):1.276-84.

36. Lobato L, Souza V, Caçador B, Soares NA, Wingester ELC, Gazzinelli MF. Efeitos de intervenção educativa na qualidade ética do consentimento livre e esclarecido. Rev. bioét. (Impr.). 2012;20(3):479-89.

37. Freire P. Pedagogia da autonomia: saberes necessários à prática educativa. 25. ed. São Paulo: Paz e Terra; 1997.

\section{Participação dos autores}

Todos os autores realizaram as etapas: trabalho de campo, concepção e projeto do estudo, análise estatística e interpretação dos dados, redação do artigo, revisão crítica relevante do conteúdo intelectual do manuscrito, aprovação final da versão a ser publicada.

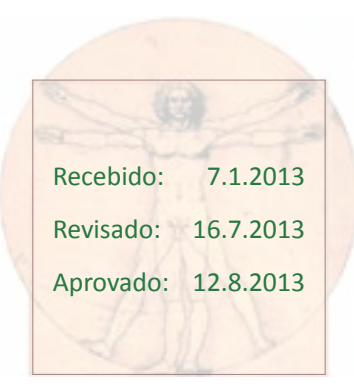

